\title{
Burkina Faso et Mali: L'excision compromet la santé des femmes
}

Frontiers in Reproductive Health

Follow this and additional works at: https://knowledgecommons.popcouncil.org/departments_sbsr-rh

Part of the Family, Life Course, and Society Commons, International Public Health Commons, and the Maternal and Child Health Commons

How does access to this work benefit you? Let us know!

\section{Recommended Citation}

"Burkina Faso et Mali: L'excision compromet la santé des femmes," FRONTIERES Résumés de Recherche Opérationnelle. Washington, DC: Population Council, 2000. 


\section{Burkina Faso L’excision compromet la Santé des et Mali Excision Femmes}

$N^{\circ} 10$

Les femmes qui, au Burkina Faso et au Mali, ont subi une excision sont plus exposées à des problèmes gynécologiques et obstétriques, notamment les saignements, des cicatrices internes, un rétrécissement vaginal, ainsi que des complications au moment de l'accouchement. Plus le type d'excision est sévère, plus le risque d'avoir d'autres problèmes de santé de la reproduction s'accrô̂t.

\section{Contexte}

En collaboration avec les Ministères de la Santé du Burkina Faso et du Mali, et l'ONG ASDAP, le Population Council a réalisé en 1998 deux études qui décrivent la survenue et la gravité des problèmes de santé liés à l'excision. Cette pratique traditionnelle implique l'ablation partielle ou totale des organes génitaux externes des filles.

Les participantes à l'étude sont des femmes venues à la formation sanitaire pour consultations prénatales, de planification familiale ou de gynécologie obstétrique, devant avoir un examen pelvien et ayant donné leur consentement. Les prestataires ont été formés à l'observation des types d'excision et des complications qui en résultent. Afin d'évaluer le rôle potentiel d'agents de changement qu'ils peuvent jouer, les prestataires du Mali ont aussi bénéficié d'une formation sur les incidences de l'excision sur la santé, ainsi que le counseling des client(e)s. Au Burkina Faso, les prestataires de services de santé ont interrogé et collecté des informations auprès de 1920 femmes dans 21 formations sanitaires des provinces rurales de Bazèga et de Zoundwèogo. Au Mali, les prestataires ont recueilli, dans les districts de Bamako et de la région de Ségou, des informations auprès de 5390 femmes dans 14 formations sanitaires urbaines et rurales.

\section{Résultats}

- La prévalence de l'excision était très élevée, 93 pourcent des clientes des formations sanitaires au Burkina Faso, et 94 pourcent de celles du Mali avaient été excisées. Au Burkina Faso le type 1 (ablation du capuchon ou de tout le clitoris) était le plus répandu. Près de trois quarts (74 pourcent) des femmes au Mali présentait le type 2 (clitoridectomie et ablation des petites lèvres). Cinq pour cent des femmes des deux groupes avaient subi la forme la plus sévère, le type 3 ou infibulation, qui consiste à l'ablation partielle ou complète des parties externes de l'organe génital avec une suture ou un rétrécissement de l'ouverture vaginale.

L'excision était pratiquée dans tous les groupes ethniques.

\section{Complications liées à l'Excision}

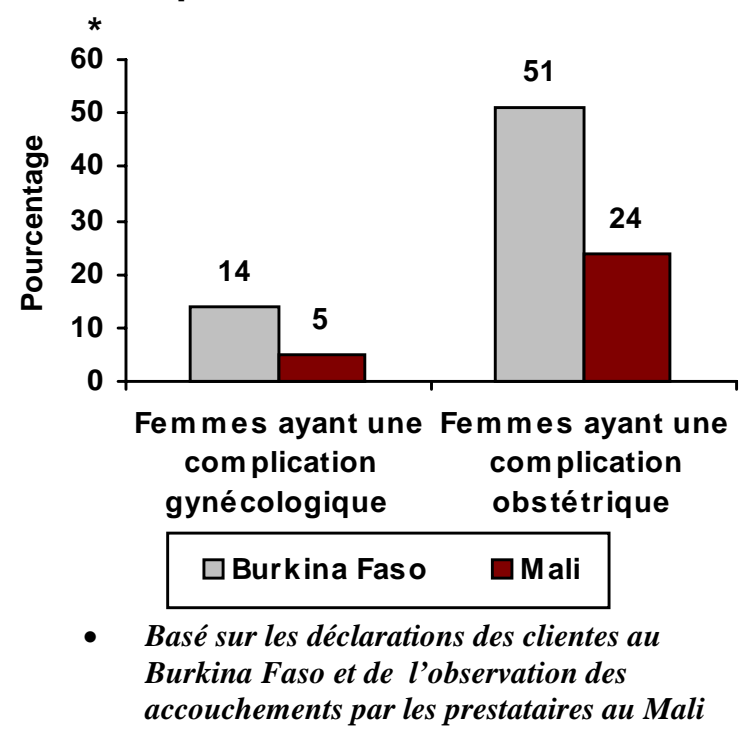


- Quatorze pourcent des clientes du Burkina Faso et 5 pourcent de celles du Mali présentaient au moins une complication gynécologique liée à l'excision. Au Burkina Faso, où la majorité des femmes a subi le type 1 , les chéloïdes et la sténose vaginale (obstruction vaginale due aux cicatrices) étaient les principales complications que présentaient les femmes. Au Mali, où le type 2 prédomine, les hémorragies étaient la complication majeure observée par le personnel, suivi des obstructions et des cicatrices chéloïdiennes.

- Dans les deux pays, les femmes qui avaient subi l'infibulation (type 3) étaient pratiquement deux fois et demie plus portées à avoir des complications gynécologiques que celles qui ont connu le type 2 . De même les femmes qui avaient subi une excision de type 1 étaient beaucoup moins exposées aux complications que celles qui ont connu des formes plus sévères.

\section{L'excision était perçue comme l'un des} principaux facteurs d'exposition à des complications durant l'accouchement, les risques augmentant selon la sévérité de l'opération. Au Burkina Faso, les femmes excisées couraient trois fois plus de risque que celles non excisées. Les femmes ayant subi le type 2 ou 3 ont également plus de chance de faire des hémorragies ou des déchirures périnéales pendant l'accouchement.

- Parmi les femmes qui, au Mali ont accouché dans une formation sanitaire, 29 pourcent de celles qui avaient été excisées avaient connu des complications durant l'accouchement comparées aux 7 pour cent de celles qui n'avaient pas été excisées. Au Mali, 5 pourcent des femmes non excisées ont des complications durant l'accouchement, comparées aux 18 pourcent des femmes qui ont subi le type $1 ; 30$ pourcent pour le type 2 et 36 pourcent pour le type 3.

- Au Burkina Faso, les femmes excisées présentaient 1,5 fois plus de risque que les femmes non excisées de voir apparaître des signes d'infections génitales, particulièrement les écoulements vaginaux ; ce qui donne à penser que l'excision prédispose les femmes aux Infections du Tractus Génital.

\section{Implications pour les programmes}

- Tous les personnels de santé doivent être informés des graves problèmes liés à l'excision.

- Outre les problèmes de droit de la Personne, les informations sur les effets néfastes de l'excision devraient être utilisées au cours des campagnes d'éducation communautaire.

- Les personnes qui assistent les femmes au moment des accouchements doivent prévoir l'éventualité d'une complication liée à l'excision.

- Diop, Nafissatou J. et al.. « Etude de l'Efficacité de la Formation du personnel socio-sanitaire dans l'éducation des client(e)s sur l'excision au Mali ». Bamako : ASDAP, DSFC et Population Council, 1998.

- Laboratoire de santé communautaire du Bazèga, Ministère de la Santé et Population Council. « Evaluation de la prévalence de la typologie, et des complications liées à l'excision chez les patientes fréquentant les formations sanitaires du Bazèga ».

Ouagadougou, Burkina Faso. 1998.

- Jones, Heidi et al. «Female Genital Cutting and its Negative Health Outcomes in Burkina Faso and Mali ». Studies in Family Planning Vol. 30, No. 3, September, 1999.

Pour de plus amples informations contacter : Population Council, 128 Sotrac Mermoz, BP : 21027, Dakar, Sénégal. Tel. : 221824-19-93 ; Fax : 221-824-19-98 ; Email : pcdakar@pcdakar.org.

Ces projets ont été réalisés avec le concours de L'AGENCE AMERICAINE POUR LE DEVELOPPEMENT INTERNATIONAL (USAID) aux termes du contrat numéro CC-3030-C-00-3008-00 et de l'accord de coopération numéro HRN-A-00-98-00012-00.

\section{(1) Popdation Cound}

